\title{
Research on the Application of Optical Illusion in Game Design
}

\author{
Yingfang Zhang ${ }^{1, a, *}$, Shiyun Li ${ }^{1, b}$ \\ ${ }^{1}$ Sino-Korean School of Multimedia Design, Shanghai University of Engineering and Science, \\ Xianxia Street, Shanghai, China \\ a zyfang0502@163.com, b 937553737@qq.com
}

Keywords: Optical illusion; Game design; Level design; Alice in Wonderland

\begin{abstract}
As a unique visual form, optical illusion can play a stunning effect in a wide range of applications, such as home design, architectural design and graphic design. The previous theoretical researches on the optical illusion are productive, and the master of art and design even makes best use of visual illusion. This paper briefly introduces the principle of visual illusion, and analyzes the classic works of visual illusion. On this basis, the optical illusion is integrated into the design of 3D maze game "Alice and Contradictory Labyrinth" for the novel visual effects and gaming experience, and a more interesting and artistic game works.
\end{abstract}

\section{Introduction}

The concept of "visual illusion" was formally introduced in the nineteenth century. Some of the Western artists including Escher and Gombrich were the leaders of this study, and had a lot of researches and experiments in this regard. Optical illusion is a peculiar but elusive visual experience embodying both strangeness and novelty. For example, there are two lines of the same length, a horizontal line and a vertical one. It is easy to believe that the vertical line is longer than the horizontal one.

Artists and designers have created a large number of classic works by blending this peculiar phenomenon into the graphic design, space design and image design.

\section{Principles of Visual Illusion}

Optical illusion refers to the graphics feel inconsistent with the facts under the interference of psychological factors or objective factors as a wrong judgment and perception is produced when people observing an object based on their own experience or improper reference.

We need to understand the formation of the vision firstly. The object reflects the light and images on the retina, and the light stimulates the photoreceptor cell on the retina to result in a nerve impulse. After transmitting to the optic centre of the cerebral cortex, the nerve impulse converts to the scene of the brain in the optic centre. Noteworthy, the generation of these scenes is processed. The image projects outward after the restoration in the optic centre, thus forming the scene people see.

In the process of visual formation, the "thinking" part of the brain is crucial, as it is not only related to the physiological sensory function, but also highly connected with the psychology. For "visual illusion", there is still no definite explanation. The well-known British biologist Crick has given three explanations, which may elaborate the optical illusion to some extent: people can easily be deceived by their own visual system; eyes provide ambiguous visual information; "see" is a building process.

Fig. 1 shows the Relativity of Maurice Cornelius Escher which is a representative classic works of optical illusion. The space built by use of the spatial logic cannot really exist in a threedimensional world, but with accurate computation and clever selected angle, its existence can be simulated by use of the optical illusion. 


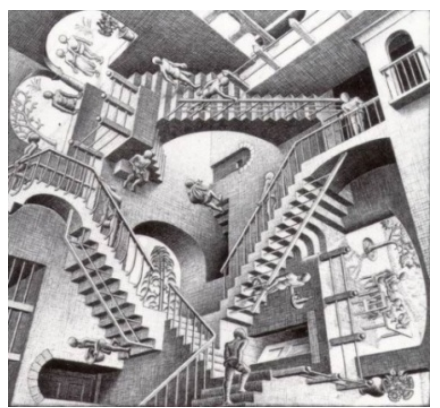

Fig. 1. The Relativity of Maurice Cornelius Escher

In real life, the graphics of optical illusion are also very common. For instance, the staggered arrangement of the black and white lattice can give a visual illusion of cross tilt (is shown in Fig. 2).

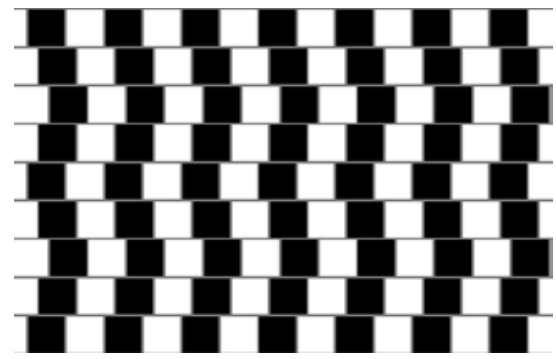

Fig. 2. The graphics of optical illusion

In recent years, the popular 3D three-dimensional painting produces results by actually using the optical illusion (is shown in Fig. 3). The artist creates the painting in a plane that looks like threedimensional and very lifelike from a specific point of view, known as three-dimensional painting, but it is only the effect that can be seen in a particular perspective after the calculation of the artist. This painting will become a bit awkward, let alone true, from other perspectives.

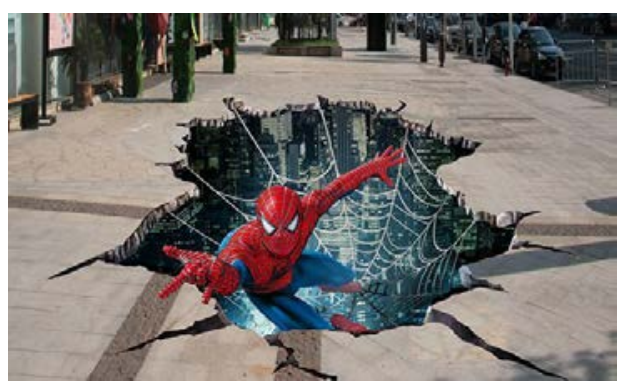

Fig. 3. The three-dimensional painting

The classic case of optical illusion used in the game is the "Monument Valley", which is a successful puzzle 3D game, and won 2014 Apple Design Award. The game was influenced by the "illusion graphics" master - Escher. Each level of the game design shows ingenuity and uses the optical illusion. The game role can walk along the wall or walk upside down; a pillar in this space is walkable, and in another space, is also walkable. However, before the appearance of the pillar, the two spaces seem to be unrelated. A variety of ingenious establishments enables the game role to walk in the unexpected space. It looks right in certain part of space, but in connection with the road taken or observation of the entire maze design, it seems incorrect, which is the contradictory space designed by use of the optical illusion. When you turn on each establishment, you can feel that the sophisticated calculation and clever conception of the producers are remarkable.

\section{Application of Optical Illusion to Alice and Contradictory Labyrinth}

Alice and Contradictory Labyrinth is a 3D maze game. The peculiar and elusive visual phenomenon of optical illusion is very consistent with the artistic conception of the literary masterpiece "Alice in Wonderland”. The combination of both presents a fancy space effect, creates 
a dreamy fairyland world and immerses players in such a game atmosphere to have a unique gaming experience.

The greatest impact of optical illusion on the game is the design of labyrinth and scene. The game consists of 4 levels. The content design of the level uses the story and plot of Alice in Wonderland as a reference blueprint, while the design of labyrinth is add the application of optical illusion in this basis.

\subsection{Application of Spatial Optical Illusion}

In the first level, the tree hole utilizes the concept of spatial optical illusion. The circular table linen on the left side of Fig.4 is actually in different horizontal planes, which players can discover by observing the right-side view. In the left-side view, the viewing angle of the game is fixed in a specific position, so that the originally staggered circular table linen looks like in the same plane, which plays the role in inducing the players. The players cannot find these table linens are at different heights unless they combine it with the right-side view. If the players only look at the leftside view, they easily walk on the "seemingly" connected circular table linen, and they will fall. Only the circular table linen actually connected is the right path.

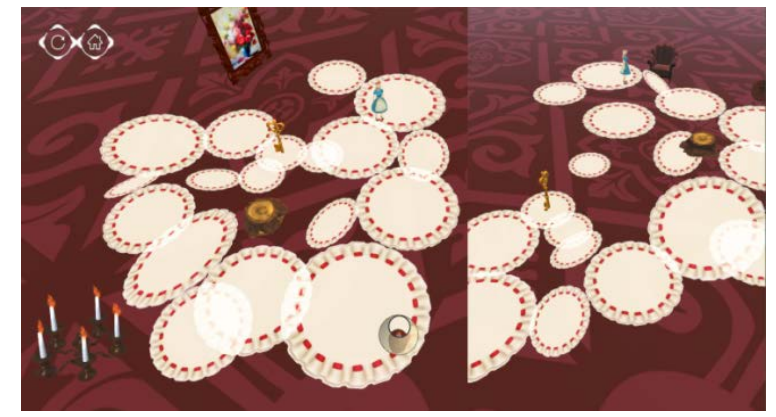

Fig.4. The first level "tree hole"

The garden in the fourth level also uses the same concept of spatial optical illusion. Differently, in the first level, the protagonist Alice is walking in the wrong perspective, with the right perspective as a reference. While in the fourth level, Alice can switch the first-person perspective and third-person perspective, but cannot see the whole picture of the labyrinth, with the upper-right map as a reference. But this map is set to be inducible in a fixed perspective. In other words, the map itself is "wrong". The road looks connected on the map which is actually disconnected or impassable.

\subsection{Application of Mirror “Optical Illusion”}

The labyrinth design of the second level "chess" applies a different concept from the first level. It designs the optical illusion with mirror, because the mirror has an effect of minifying and extending the space. The labyrinth design of this level also uses the wonderful effect of specular reflection. In a white-black and high-low staggered space, the effect of specular reflection add even more traces of psychedelic feeling to the originally minimalist scene. In addition, the specular reflection also extends the space, so that the space looks more spacious. The background outside the labyrinth is designed to be white and add a sense of wide open space. Thanks to the design of open scene and the effect of specular reflection, the player is easy to visually judge this space is large or the labyrinth is very complicated. However, this is, in fact, not true, because the scene is a labyrinth that the players can walk out within 90 seconds (is shown in Fig. 5). 


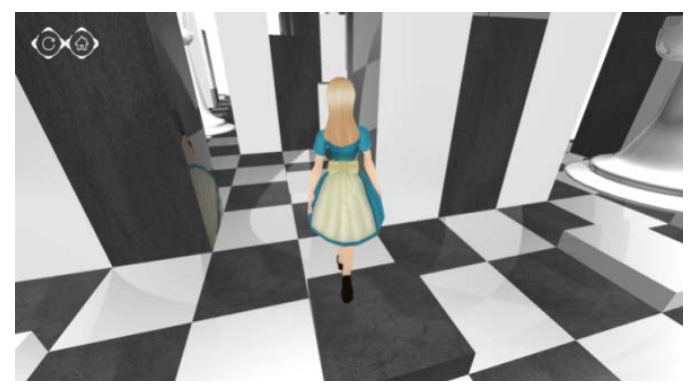

Fig. 5. The second level "chess"

\subsection{Application of Bounded and Unbounded Concept}

The scene design of the third level "poker" uses the bounded and unbounded concept in the works of Escher. Escher's many works take advantage of the mathematical concepts such as circulation and fractal. The seemingly limited content can be continuously extended with fractal. The vertex seemingly exists but you can never reach it. This is an amazing point of Escher's works. This concept is applied to this level. The scene is designed to be black. Players will be unable to distinguish the direction in the dark and have no idea about the border. The players need to keep walking to avoid the capture of the poker soldiers. And this seemingly unbounded space is essentially bounded, with fire as its border. The seemingly unbounded is bounded, and the black space constantly extends outward, which makes the bounded space to be unbounded.

This is the most difficult level in the game. In such a dark scene, it is difficult for players to determine the direction. Players may walk back and forth in the scene without finding the exit. After the time limit of 60 seconds, the game automatically ends, or if Alice is caught up by the poker soldiers, the game also ends.

\section{Conclusion}

Overall, the impact of the optical illusion on the gaming experience is to bring players with a sense of novelty. Adding an operationally or visually brand new content to 3D game arouse the interest of players in game and produces a good gaming experience.

Each level of this game displays different content, by the uses of different elements and details of Alice in Wonderland. The application and performance of optical illusion in each level is also somewhat varied, which tries some new method of game play and pattern of manifestation. The downside is that, the manifestation of optical illusion is still insufficient, and the best effect of optical illusion is not given play. The details and elements of Alice in Wonderland is referred, but the communication of the story is not very clear.

\section{References}

[1] Ruan Lige. Introduction to Optical Illusion [J]. Literature Life, 2013(9):195-195

[2] Hu Yuling. On the Application of Optical Illusion to Artistic Creation of Contemporary Public Space [J]. Art Education Research, 2013(07):62-63

[3] Li Xinyu. Research on the Application of Optical Illusion in Visual Communication Design[D]. Henan University, 2012

[4] Guo Mengqi. Over Shoes Over Boots - Research on Application of Optical Illusion to Interactive Interface Design[D]. Beijing Institute of Graphic Communication, 2014

[5] Hu Yanxia. Analysis on Graphic Expression Language of Optical Illusion in Shigeo Fukuda's Posters [D]. Beijing Jiaotong University, 2012

[6] Feng Tao. The Expression Method and Application of Dynamic Optical Illusion [D]. Soochow University, 2014 
[7] Zhang Min. Design and Implementation of Game Based on Unity 3D[J]. Computer CD Software and Applications, 2014(18):269-270 